\title{
Hypocholesterolaemic effect of whole-grain highland hull-less barley in rats fed a high-fat diet
}

\author{
Xuejuan Xia ${ }^{1}$, Guannan $\mathrm{Li}^{2}$, Jiaxin Song ${ }^{1}$, Jiong Zheng ${ }^{1}$ and Jianquan $\mathrm{Kan}^{1,3 *}$ \\ ${ }^{1}$ College of Food Science, Southwest University, Chongqing 400715, People's Republic of China \\ ${ }^{2}$ College of Biotechnology, Southwest University, Chongqing 400715, People's Republic of China \\ ${ }^{3}$ Laboratory of Quality \& Safety Risk Assessment for Agro-products on Storage and Preservation (Chongqing), Ministry of \\ Agriculture, 400715, People's Republic of China
}

(Submitted 27 September 2017 - Final revision received 21 February 2018 - Accepted 2 March 2018)

\section{Abstract}

Whole-grain highland hull-less barley (WHLB) contains high amounts of bioactive compounds that potentially exhibit cholesterol-lowering effects. This study investigated the hypocholesterolaemic effect of WHLB. A total of seventy-two male Sprague-Dawley rats were divided into four groups and were fed with the normal control diet, high-fat diet (HFD) and HFD containing low or high dose (10 or $48.95 \%)$ of WHLB. High dose of WHLB significantly decreased the organ indexes of liver and abdominal fat and lipid levels of plasma and liver in HFD rats. The lipid regulation effect of WHLB, which was reconfirmed through hepatocyte morphologic observation, was accompanied by a large excretion of bile acids in the small intestinal contents and the faeces. Real-time PCR analyses, which were further reconfirmed through Western blot analyses, revealed that a high dose of WHLB significantly enhanced the hepatic expressions of AMP-activated protein kinase $\alpha$, cholesterol $7 \alpha$-hydroxylase, LDL receptor, liver X receptor, and PPAR $\alpha$ and decreased the expression of 3-hydroxy-3-methylglutaryl coenzyme A reductase. It also enhanced the ileal expression of farnesoid X receptor and resulted in the decrease of expression of apical sodiumdependent bile acid transporter. WHLB exhibited hypocholesterolaemic effects mainly by inhibiting cholesterol synthesis, cholesterol accumulation in peripheral tissue, and bile acid reabsorption and by stimulating bile acid synthesis.

\section{Key words: Hull-less barley: Cholesterol metabolism: Cellular biomarkers: Gene expression: Dietary fibres}

Cholesterol is a lipid that is synthesised in the body. It is also consumed as part of the daily diet. Although cholesterol plays important biochemical roles in the body, high cholesterol levels and abnormal metabolism can lead to the development of chronic obesity-related diseases, such as $\mathrm{CVD}^{(1,2)}$. As an alternative to pharmacological medicine, dietary means to control risk factors for obesity-related diseases have recently received considerable attention to reduce CVD risk ${ }^{(3)}$. Accumulated evidence suggests that the cholesterol-lowering effects could be achieved by the following mechanisms, each with its own cellular markers: inhibition of hepatic synthesis, which has AMP-activated protein kinase $\alpha(\mathrm{AMPK} \alpha)^{(4)}$, 3-hydroxy3-methylglutaryl coenzyme A reductase (HMG-CoAr) ${ }^{(5)}$ and sterol regulatory element-binding protein-1c (SREBP-1C) ${ }^{(6)}$ as biomarkers; inhibition of cholesterol accumulation in peripheral tissue, with $\operatorname{apoB}^{(7)}$, LDL receptor $(\mathrm{LDLR})^{(8,9)}$, liver $\mathrm{X}$ receptor $(\mathrm{LXR})^{(10)}$ and PPAR $\alpha^{(11)}$ as biomarkers; acceleration of cholesterol conversion into bile acids, with cholesterol $7 \alpha$-hydroxylase
$(\mathrm{CYP7A1})^{(11)}$ and farnesoid X receptor (FXR) ${ }^{(12)}$ as biomarkers; and inhibition of bile acid reabsorption, with apical sodiumdependent bile acid transporter (ASBT), FXR and ileal bile acid-binding protein (IBABP) ${ }^{(13)}$ as biomarkers. The relative activity of each mechanism can be assessed by quantifying relevant biomarkers ${ }^{(3)}$.

Increasing evidence supports that higher whole-grain consumption is associated with a reduced risk of CVD development ${ }^{(14,15)}$. According to previous studies, barley exhibited hypocholesterolaemic effects, which may be attributed to its bioactive components, particularly dietary fibre and $\beta$-glucans ${ }^{(15-19)}$. In contrast to regular hulled barley, hull-less barley is more advantageous in terms of processing and food applications $^{(20)}$. Highland hull-less barley, also known as Qingke in Chinese, grows under extreme geographical conditions and contains a higher amount of $\beta$-glucans and dietary fibre ${ }^{(21)}$. However, available information on the cholesterollowering effect of whole-grain highland hull-less barley

Abbreviations: AMPK $\alpha$, AMP-activated protein kinase $\alpha$; ASBT, apical sodium-dependent bile acid transporter; BC, blank control; CYP7A1, cholesterol 7 $\alpha$ hydroxylase; FXR, farnesoid X receptor; HD, high dose; HFD, high-fat diet; HMG-CoAr, 3-hydroxy-3-methylglutaryl coenzyme A reductase; IBABP, ileal bile acid-binding protein; LD, low dose; LDLR, LDL receptor; LXR, liver X receptor; NC, normal control; SREBP-1c, sterol regulatory element-binding protein-1c; TC, total cholesterol; WHLB, whole-grain highland hull-less barley.

* Corresponding author: J. Kan, fax +86 682519 47, email kanjianquan@163.com 
(WHLB) is limited. In particular, the specific mechanism by which WHLB affects cholesterol metabolism is still unknown.

Thus, the present study aims to determine the hypocholesterolaemic effect and mechanism of WHLB. Plasma levels of total cholesterol (TC), TAG, LDL-cholesterol and HDLcholesterol and TC, TAG and total lipid levels of the liver were investigated after high-fat diet (HFD)-fed rats were given different amounts of WHLB. The degrees of hepatic steatosis were examined through haematoxylin-eosin (H\&E) staining of sections of the liver. Bile acid levels in small intestinal contents and faeces were examined simultaneously. To determine the molecular mechanism, real-time PCR (RT-PCR) was conducted to quantify the mRNA levels of abovementioned cellular biomarkers. Protein levels of CYP7A1, HMG-CoAr, LDLR, ASBT and IBABP were analysed to confirm the main results of RT-PCR. Overall, this study may provide important implications for future functional food development of WHLB.

\section{Methods}

\section{Sample preparation}

WHLB (Tibet Hordeum vulgare L. Zangqing 320) was provided by Jun Pro Food Co., Ltd. WHLB was dried in an oven at $55^{\circ} \mathrm{C}$ for $24 \mathrm{~h}$ and then ground and passed through an 80-mesh sieve $(0.5 \mathrm{~mm})$. The moisture (American Association of Cereal Chemists (AACC) method 44-16) ${ }^{(22)}$ content in WHLB was 8.71 (SD 0.03)\%. The contents (based on dry weight) of ash (AACC method 08-01) ${ }^{(22)}$, fat (AACC method 30-10) ${ }^{(22)}$, protein (KjelFlex K-360 nitrogen determination system; Buchi Laboratory Equipment Trading, Ltd) and $\beta$-glucans (Mixed-linkage $\beta$-glucan kit; Megazyme Int. Ireland Ltd) in WHLB were 1.95 (sD 0.08), 1.03 (sD 0.02), 17.00 (sD 0.26) and 5.77 (sD 0.28) g/100 g, respectively. The contents (based on dry weight) of total dietary fibre, insoluble dietary fibre, and soluble dietary fibre contents (AOAC method $991.43)^{(23)}$ in WHLB were 19.01 (SD 0.54), 11.64 (SD 0.95) and 9.28 (sD 0.01) g/100 g, respectively.

\section{Animals and diets}

Four dietary groups were created to investigate the dosage effect of WHLB, as follows: normal control (NC) group, fed with normal AIN-93G ${ }^{(24)}$ diet; blank control (BC) group, fed with an HFD, plus $1 \%$ cholesterol and with additional $10 \%$ lard instead of soyabean oil ${ }^{(10)}$; the low-dose (LD) group, fed with HFD containing low-dose (10\%) of WHLB; and the high-dose (HD) group, fed with HFD containing high dose (48.95\%) of WHLB instead of maize $\operatorname{starch}^{(21)}$ (online Supplementary Table S1). To study the time effect of WHLB, half of the animals in each group were fed with experimental diets for 4 weeks, whereas the other half was fed for 8 weeks. Therefore, a total of eight groups were created. The sample size was calculated by power analysis according to the formula for quantitative data published by Charan \& Kantharia ${ }^{(25)}$. The type I error $(\alpha /$ significance level $)$ was set at 0.05 and the power at 0.80 (with $\beta$ /type II error at 0.02 ). The effect size was set at 0.50 (plasma TC of rats decreased from 4.00 to $3.50 \mathrm{mmol} / 1$ after high diet dose of WHLB) and sD at $0.33(\mathrm{mmol} / \mathrm{l})$, based on an initial pilot study. A sample size of eight animals for each group was sufficient for consideration of $10 \%$ attrition. We finally used nine rats per group, because three rats were housed in one stainless steel screen-bottomed cage because of the limited space. Therefore, a total of 72 4-week-old specific pathogen-free SpragueDawley male rats (151 (sD 12) g weight) were purchased from Chongqing Tengxin Biotechnology Co., Ltd (permitted by SCXK 2012-0005 (Chongqing)). The room was illuminated with a $12 \mathrm{~h}$ light-12 h dark cycle at a constant temperature of $23 \pm 2^{\circ} \mathrm{C}$ and a relative humidity of 55 (SD 10)\%. The rats were acclimated by feeding with an AIN-93G diet for 1 week and were given free access to food and water. After acclimation, rats were randomly assigned to the four dietary groups ( $n$ 18/group). The experiment design was approved by the Animal Care and Use Committee of Southwest University (Permit SYXK2009-0002) and strictly conducted in accordance with the guidelines for animal care of the National Institute of Health ${ }^{(26)}$.

\section{Sampling and analytical procedures}

Fresh faeces (approximately $0 \cdot 2-1 \cdot 0 \mathrm{~g}$ and $0 \cdot 2-0.4 \mathrm{~g}$ ) of each rat were collected weekly. The water content and $\mathrm{pH}$ of the faeces were determined. Faeces were collected on the last $3 \mathrm{~d}$ of the experimental period, freeze-dried, and then milled for bile acid measurement ${ }^{(27)}$. On the last day of the experimental period, the rats were fasted overnight (12-14h) and lightly anaesthetised with diethyl ether ${ }^{(28)}$. Their body weights were measured. After decapitation, blood was collected from the neck of each rat into a blood collection tube (Vacutainer; Liuyang City Medical Instrument Factory) containing heparin as an anticoagulant. The plasma was centrifuged at $1400 \boldsymbol{g}$ at $4^{\circ} \mathrm{C}(5810$ centrifuge; Eppendorf China Ltd) for $15 \mathrm{~min}$ and the obtained plasma was stored at $-80^{\circ} \mathrm{C}$ until analysis.

The stomach, liver, kidney, abdominal fat and caecum (together with the contents) of each rat were immediately removed, washed with ice-cold $0.9 \% \mathrm{NaCl}$ solution, blotted dry on filter paper, and weighed. Organ index was evaluated as follows: organ weight $(\mathrm{g}) /$ body weight $(\mathrm{g}) \times 100^{(10)}$. The liver was incised to $5 \times 5 \times 5 \mathrm{~mm}$ sections and fixed in Bouin solution $^{(3)}$. Liver tissues $(1.0$ and $0.1 \mathrm{~g})$ from each rat were dissected, washed, frozen in liquid $\mathrm{N}_{2}$, and stored at $-80^{\circ} \mathrm{C}$ for lipid analysis and RNA extraction, respectively. The small intestine of each rat was excised. The small intestine was douched with ice-cold $0.9 \% \mathrm{NaCl}$ solution. Then, a suspension of intestinal content was collect, freeze-dried, weighed, and then milled for bile acid measurement ${ }^{(29)}$. A total of $0 \cdot 1 \mathrm{~g}$ of the small intestine (ileum part) in each rat was dissected, washed, frozen in liquid $\mathrm{N}_{2}$, and stored at $-80^{\circ} \mathrm{C}$ for RNA extraction. Liver and ileum tissues $(0 \cdot 2 \mathrm{~g})$ were collected from each rat in the NC, BC and HD groups after 8 weeks of feeding and stored at $-80^{\circ} \mathrm{C}$ for protein extraction.

\section{Lipid analysis}

The concentrations of bile acids in freeze-dried small intestinal contents and faeces were determined using a Rat Total Bile Acid Elisa kit (Fengxiang Biotech Co., Ltd) according to the manufacturer's instructions. Total lipid in the liver was extracted according to the method of Folch et al $^{(30)}$ and subsequently determined gravimetrically ${ }^{(27)}$. The levels of TAG and TC in the 
plasma and liver were quantified with enzymatic methods using assay kits purchased from Sichuan Maker Biotechnology Co., Ltd. LDL-cholesterol and HDL-cholesterol in the plasma were also quantified through enzymatic methods using assay kits (Sichuan Maker). Measurements were performed using a 7020 Automatic Analyzer (Hitachi) in accordance with the manufacturer's instructions.

\section{Histopathological analysis}

The liver was stored in $70 \%$ ethanol until histological analysis after storing at Bouin solution for $24 \mathrm{~h}$. A portion of the stored liver was embedded in paraffin and then cut into $5-\mu \mathrm{m}$ thick semi-serial histological sections using RM2235 microtome (Microsystems $\mathrm{GmbH}$ ). Then H\&E staining was performed ${ }^{(3)}$. Representative images were acquired at $20 \times$ magnification using an Olympus BX43 microscope (Olympus Optical Co.).

\section{RNA extraction and real-time PCR analysis of gene expression}

Total RNA was extracted from the frozen liver and ileum samples using RNAiso Plus reagent (Takara Bio, Inc.). RNA concentration and quality were checked using a NanoDrop 2000 spectrophotometer (Thermo Scientific and integrity was examined using a $1.2 \%(\mathrm{w} / \mathrm{v})$ agarose gel. Extracted RNA was used for complementary DNA (cDNA) synthesis using PrimeScript RT reagent kit with gDNA Eraser (Takara). The resulting cDNA was amplified using the SYBR Premix Ex Taq II (Takara) and specific primers (Table 1, synthesised by Sangon Biotech Co., Ltd). The gene expressions of AMPK $\alpha$, ApoB, CYP7A1, FXR, HMG-COAr, LDLR, LXR, PPAR $\alpha$ and SREBP-1c in the liver and ASBT, FXR, and IBABP in the ileum were investigated. RT-PCR was performed using the LightScanner 32 system (Biofire Diagnostics). The amplification programme consisted of one cycle of $95^{\circ} \mathrm{C}$ for $30 \mathrm{~s}, 45-55$ cycles of $95^{\circ} \mathrm{C}$ for $5 \mathrm{~s}$ and $60^{\circ} \mathrm{C}$ for $20 \mathrm{~s}$. To examine the quality of amplification products, melting curve analysis was performed under the following conditions: $95^{\circ} \mathrm{C} 0 \mathrm{~s}, 20^{\circ} \mathrm{C} / \mathrm{s} ; 65^{\circ} \mathrm{C} 15 \mathrm{~s}, 20^{\circ} \mathrm{C} / \mathrm{s} ; 95^{\circ} \mathrm{C} 0 \mathrm{~s}, 0.1{ }^{\circ} \mathrm{C} / \mathrm{s}$, continuous measurement of fluorescence; and $50^{\circ} \mathrm{C}$ cooled $30 \mathrm{~s}$, $20^{\circ} \mathrm{C} / \mathrm{s}$. Melting peaks were recorded by plotting the absolute value of the first derivative against the temperature ${ }^{(32)}$. Gene expression was calculated using $2^{-\Delta \Delta C_{t}}$ method; the $\beta$-actin gene was used as the housekeeping gene for normalisation ${ }^{(31)}$.

\section{Protein extraction and Western blot assays}

Protein was extracted from the frozen liver and ileum samples using a commercial Tissue Total Protein Lysis Buffer (Biotech). The amount of pooled proteins was determined using a commercial Bradford Protein Assay Kit (Beijing Solarbio Science \& Technology Co., Ltd) with bovine serum albumin as the standard. SDS-PAGE and Western blot analysis were performed according to the protocol of Zhang et al. ${ }^{(3)}$ After blocking in PBS containing $2 \%$ Tween- 20 and $3 \%$ bovine serum antigen, the membranes were incubated with anti-CYP7A1, anti-HMGCoAr, anti-LDLR, anti-ASBT or anti-IBABP experimental antibodies (Abcam, Inc.) overnight at $4^{\circ} \mathrm{C}$. The $\beta$-actin was used as a loading control. The membrane was washed thrice with PBS-T (0.1\% Tween-20 in PBS) and incubated with horseradish peroxidase-conjugated secondary antibodies at room temperature for $1 \mathrm{~h}$. Reactive bands were visualised by a SuperSignal $\mathrm{R}$ West Pico Chemiluminescence substrate (Pierce). The density of each protein immunoblot was determined using Quantity One Analyzer software (Bio-Rad Laboratories).

\section{Statistical analysis}

The results are reported as mean values and standard deviations. Statistical analysis was conducted through one-way ANOVA using SPSS 20.0 software (IBM). $P<0.05$ was regarded as statistically significant.

\section{Results}

Organ indexes

The changes in organ indexes among all groups are displayed in Table 2. No significant differences in stomach and kidney indexes were observed among all groups. The indexes of liver and abdominal fat of the $\mathrm{BC}$ group were significantly higher than those in the NC group. The abdominal fat indexes in the LD group were significantly lower, whereas the caecum indexes

Table 1. Primers for real-time PCR

\begin{tabular}{|c|c|c|c|c|}
\hline Detected sites & Gene & Forward primer & Reverse primer & Reference \\
\hline \multirow[t]{8}{*}{ Liver } & AMPKa & 5'-ACCTGAGAACGTCCTGCTTG-3' & 5'-GGCCTGCGTACAATCTTCCT-3' & (7) \\
\hline & ApoB & 5'-TTGACACACTGAAGTTCCTA-3' & 5'-ACATCAAACCCTGGTATTAG-3' & (29) \\
\hline & CYP7A1 & 5'-GAGGGATTGAAGCACAAGAACC-3' & 5'-ATGCCCAGAGAATAGCGAGGT-3' & (27) \\
\hline & HMG-CoAr & 5'-GACCAACCTTCTACCTCAGCAAG-3' & 5'-ACAACTCACCAGCCATCACAGT-3' & (27) \\
\hline & LDLR & 5'-CAGCTCTGTGTGAACCTGGA-3' & 5'-TTCTTCAGGTTGGGGATCAG-3' & (31) \\
\hline & LXR & 5'-CATCAAGGGAGCACGCTACATT-3' & 5'-GCATTTGCGAAGGCGACAC-3' & (31) \\
\hline & PPAR $a$ & 5'-TCACACAATGCAATCCGTTT-3' & 5'-GGCCTTGACCTTTGTTCATGT-3' & (31) \\
\hline & SREBP-1C & 5'-GGAGCCATGGATTGCACATT-3' & 5'-AGGAAGGCTTCCAGAGAGGA-3' & \\
\hline \multirow[t]{2}{*}{ Ileum } & ASBT & 5'-GTGACATGGACCTCAGTGTTAGC-3' & 5'-GTAGGGGATCACAATCGTTCCT-3' & (27) \\
\hline & IBABP & 5'-CAGACTTCСССААСТАTCACCAG-3' & 5'-TCAAGCCACCСТCTTGCTTAC-3' & (27) \\
\hline Liver and ileum & FXR & 5'-GCTAAGGAAGTGCAGAGAGATGG-3' & 5'-ATAGCTTGGTCGTGGAGGTCACT-3' & (31) \\
\hline Housekeeping gene & $\beta$-Actin & 5'-ACGGTCAGGTCATCACTATCG-3' & 5'-GGCATAGAGGTCTTTACGGATG-3' & (27) \\
\hline
\end{tabular}

AMPKa, AMP-activated protein kinase $a$; CYP7A1, cholesterol 7a-hydroxylase; HMG-CoAr, 3-hydroxy-3-methylglutaryl coenzyme A reductase; LDLR LDL receptor; LXR, liver X receptor; SREBP-1c, sterol regulatory element-binding protein-1c; ASBT, apical sodium-dependent bile acid transporter; IBABP, ileal bile acid-binding protein; FXR, farnesoid $X$ receptor. 
Table 2. Effect of diet with different doses of whole-grain highland hull-less barley on organ indexes of high-fat diet Sprague-Dawley rats* (Mean values and standard deviations; $n$ 9)

\begin{tabular}{|c|c|c|c|c|c|c|c|c|}
\hline \multirow[b]{2}{*}{ Organ indexes } & \multicolumn{2}{|c|}{$\mathrm{NC}$} & \multicolumn{2}{|c|}{$\mathrm{BC}$} & \multicolumn{2}{|c|}{ LD } & \multicolumn{2}{|c|}{$\mathrm{HD}$} \\
\hline & Mean & SD & Mean & SD & Mean & SD & Mean & SD \\
\hline \multicolumn{9}{|l|}{ Stomach index } \\
\hline Week 4 & $0.56^{\mathrm{a}}$ & 0.02 & $0.59^{a}$ & 0.16 & $0.55^{a}$ & 0.13 & $0.51^{a}$ & 0.02 \\
\hline Week 8 & $0.44^{\mathrm{a}}$ & 0.03 & $0.47^{a}$ & 0.03 & $0.43^{\mathrm{a}}$ & 0.04 & $0.46^{\mathrm{a}}$ & 0.07 \\
\hline \multicolumn{9}{|l|}{ Liver index } \\
\hline Week 4 & $2.97^{\mathrm{a}}$ & 0.05 & $3.27^{b}$ & 0.29 & $3.11^{a}$ & 0.37 & $3.03^{a}$ & 0.21 \\
\hline Week 8 & $2.99^{\mathrm{a}}$ & 0.052 & $3 \cdot 87^{b}$ & 0.15 & $3.81^{\mathrm{b}}$ & 0.22 & $3.48^{\mathrm{c}}$ & 0.45 \\
\hline \multicolumn{9}{|l|}{ Kidney index } \\
\hline Week 4 & $0.75^{\mathrm{a}}$ & 0.08 & $0.71^{\mathrm{a}}$ & 0.06 & $0.77^{a}$ & 0.03 & $0.75^{a}$ & 0.02 \\
\hline Week 8 & $0.75^{a}$ & 0.02 & $0.71^{\mathrm{a}}$ & 0.01 & $0.77^{a}$ & 0.05 & $0.75^{a}$ & 0.08 \\
\hline \multicolumn{9}{|c|}{ Abdominal fat index } \\
\hline Week 4 & $0.66^{a}$ & 0.15 & $1.02^{\mathrm{b}}$ & 0.08 & $0.76^{\mathrm{a}}$ & 0.06 & $0.59^{\mathrm{a}}$ & 0.14 \\
\hline Week 8 & $0.71^{a}$ & 0.18 & $1.09^{\mathrm{b}}$ & 0.14 & $0.85^{a, b}$ & 0.07 & $0.69^{\mathrm{a}}$ & 0.19 \\
\hline \multicolumn{9}{|l|}{ Caecum index } \\
\hline Week 4 & $1 \cdot 41^{\mathrm{a}}$ & 0.31 & $1.56^{a}$ & 0.15 & $1 \cdot 74^{b}$ & 0.37 & $2 \cdot 32^{\mathrm{c}}$ & 0.31 \\
\hline Week 8 & $1.42^{\mathrm{a}}$ & 0.29 & $1 \cdot 25^{\mathrm{a}}$ & 0.02 & $1.28^{\mathrm{a}}$ & 0.27 & $2 \cdot 10^{\mathrm{b}}$ & 0.31 \\
\hline
\end{tabular}

were significantly higher than those in the $\mathrm{BC}$ group after 4 -week feeding. All organ indexes of the LD group were similar with those of the BC group after 8-week feeding. However, the indexes of liver and abdominal fat of HD groups were significantly lower than those in the $\mathrm{BC}$ group. Caecum indexes of $\mathrm{HD}$ groups were significantly higher than those in other groups.

\section{Plasma and liver lipid profiles}

Changes in plasma and liver lipid levels are shown in Fig. 1. The plasma TC, TAG, HDL-cholesterol and LDL-cholesterol levels in the $\mathrm{BC}$ group were significantly higher than those in the $\mathrm{NC}$ group. The TC and LDL-cholesterol levels in the HD group were significantly lower than those in the $\mathrm{BC}$ group. HDL-cholesterol levels in the HD group were significantly higher than those in the BC group after 8-week feeding. The TC, TAG, and total lipid levels in the liver of all HFD groups were significantly higher than those in the NC group. The TC content and lipid concentration in the liver of the HD group were significantly lower than those in the BC group at week 8. TAG levels of the HD group were slightly lower than the $\mathrm{BC}$ and $\mathrm{LD}$ groups, whereas the difference was not significant. The lipid levels of the LD group showed no significant differences with those of the $\mathrm{BC}$ group.

\section{Haematoxylin-eosin-stained sections of liver}

After stained with H\&E, the degrees of hepatic steatosis were examined (Fig. 2). In the liver of the NC group, the cells had clear borders with intact cytoplasm and prominent nuclei. Moreover, no inflammatory cell infiltrate was observed. Different degrees of hepatic steatosis were observed in other rat groups. Significant morphological changes were observed in the liver cells of the $\mathrm{BC}$ group after 4 weeks of the HFD. Steatosis became significant, excessive lipid accumulation occurred in vesicles, and most lipid droplets surrounded the nucleus or were located at the side
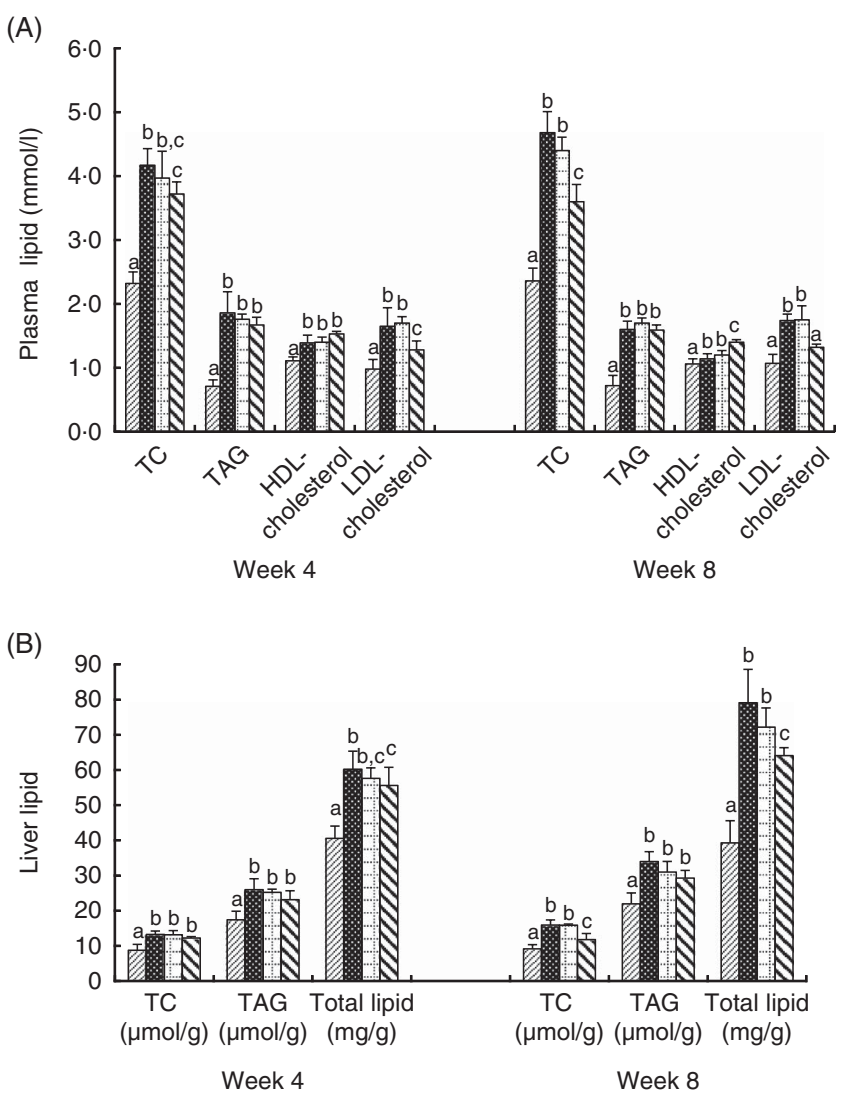

Fig. 1. Effect of diet with different doses of whole-grain highland hull-less barley on plasma (A) and liver (B) lipid levels of high-fat diet Sprague-Dawley rats. Values are means $(n 9)$ and standard deviations. ${ }^{\text {a,b,c }}$ Mean values of the same index in the four groups with unlike letters are significantly different $(P<0.05)$. TC, total cholesterol; $\Theta$, normal control group; $\mathbf{A}$, blank control group; $\square$, low-dose group; $\square$, high-dose group.

of the cells. Meanwhile, some lipids formed large droplets after 8 weeks of the HFD. Compared with the BC group, hepatic steatosis slightly decreased in the LD group, whereas remarkably decreased in the HD group.

\section{Bile acid levels in small intestinal contents and faeces}

The dry weights of small intestinal contents and faeces were not affected by fat intake (Table 3). The dry weights of faeces in the $\mathrm{HD}$ group were significantly higher than other groups. The bile acid levels of small intestinal contents and faeces in the BC and LD groups showed no significant difference after 8-week feeding. However, the bile acid levels in small intestinal contents and faeces of HD groups were significantly higher than those of the other groups. Changes in water content and $\mathrm{pH}$ of faeces were also measured (online Supplementary Fig. S1). The faecal water contents of all groups were fluctuating changed. Similarly to water content, the faecal $\mathrm{pH}$ of all groups fluctuated. However, the $\mathrm{pH}$ of the HD group had the lowest values compared with other groups.

\section{mRNA levels of key genes in hepatic and ileal lipid metabolism}

The integrity of the total RNA extracted from liver was verified by agarose gel electrophoresis images, in which three clear 

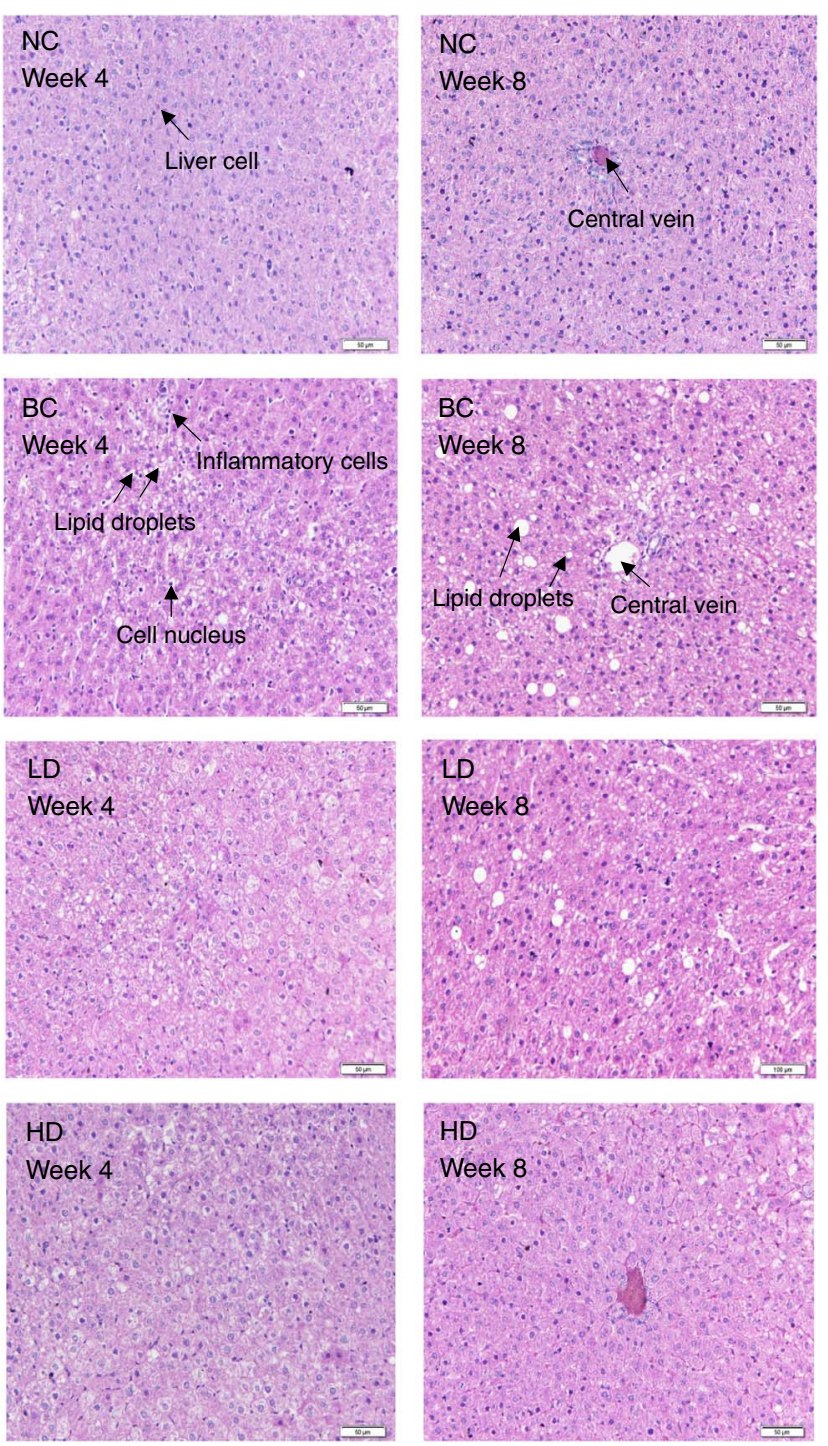

Fig. 2. Effect of diet with different doses of whole-grain highland hull-less barley on haematoxylin-eosin-stained liver sections of high-fat diet SpragueDawley rats. NC, normal control group; BC, blank control group; LD, low-dose group; HD, high-dose group.

bands (5S, $18 \mathrm{~S}$ and 28S) were observed for each representative sample (online Supplementary Fig. S2). The specificity of primers was confirmed by melting curves, in which only one dominant peak was observed for each primer (online Supplementary Fig. S3). Changes in mRNA levels of measured genes are shown in Fig. 3. The mRNA levels of ApoB, SREBP-1c and HMG-CoAr in the BC group were significantly higher than those in the NC group. The mRNA levels of measured genes in the LD group showed no significant difference with that in the $\mathrm{BC}$ group, except for PPAR $\alpha$ levels which were significantly higher than those in the $\mathrm{BC}$ group after 4-week feeding. Compared with the $\mathrm{BC}$ group, the mRNA levels of AMPK $\alpha$, LDLR, PPAR $\alpha$ and ileal FXR in the HD group were significantly higher, whereas mRNA levels of HMG-CoAr and ASBT were significantly lower. The mRNA levels of CYP7A1 and LXR in the HD group were significantly higher than those in the $\mathrm{BC}$ group after 8-week feeding.
Table 3. Effect of diet with different doses of whole-grain highland hullless barley on the bile acid levels in small intestinal contents and faeces of high-fat diet Sprague-Dawley rats

(Mean values and standard deviations; $n$ 9)

\begin{tabular}{|c|c|c|c|c|c|c|c|c|}
\hline & \multicolumn{2}{|c|}{ NC } & \multicolumn{2}{|c|}{$\mathrm{BC}$} & \multicolumn{2}{|c|}{ LD } & \multicolumn{2}{|c|}{ HD } \\
\hline & Mean & SD & Mean & SD & Mean & SD & Mean & SD \\
\hline \multicolumn{9}{|c|}{ Small intestinal content } \\
\hline \multicolumn{9}{|c|}{ Dry weight $(\mathrm{g})$} \\
\hline Week 4 & $0.38^{a}$ & 0.05 & $0.34^{a}$ & 0.05 & $0.35^{a}$ & 0.04 & $0.34^{a}$ & 0.05 \\
\hline Week 8 & $0.35^{a}$ & 0.02 & $0.30^{\mathrm{a}}$ & 0.02 & $0.32^{a}$ & 0.01 & $0.36^{a}$ & 0.05 \\
\hline \multicolumn{9}{|c|}{ Bile acid $(\mu \mathrm{mol} / \mathrm{g})$} \\
\hline Week 4 & $44 \cdot 61^{a}$ & $4 \cdot 29$ & $65.63^{b}$ & $3 \cdot 70$ & $76 \cdot 48^{c}$ & 1.64 & $83.76^{d}$ & 3.49 \\
\hline Week 8 & $46 \cdot 11^{a}$ & $3 \cdot 20$ & $66 \cdot 11^{\mathrm{b}}$ & $5 \cdot 24$ & $74 \cdot 24^{b}$ & $5 \cdot 29$ & $91.99^{C}$ & $2 \cdot 12$ \\
\hline \multicolumn{9}{|c|}{ Faeces } \\
\hline \multicolumn{9}{|c|}{ Dry weight (g/d) } \\
\hline Week 4 & $1.08^{a}$ & 0.07 & $1 \cdot 22^{a}$ & 0.11 & $1 \cdot 32^{\mathrm{a}}$ & 0.12 & $1 \cdot 81^{b}$ & 0.10 \\
\hline Week 8 & $1 \cdot 11^{a}$ & 0.05 & $1 \cdot 30^{\mathrm{a}}$ & 0.07 & $1.41^{a}$ & $0 \cdot 11$ & $2 \cdot 16^{b}$ & 0.08 \\
\hline \multicolumn{9}{|c|}{ Bile acid $(\mu \mathrm{mol} / \mathrm{d})$} \\
\hline Week 4 & $11.63^{a}$ & 0.24 & $18 \cdot 21^{\mathrm{b}}$ & 2.63 & $20 \cdot 55^{\mathrm{b}}$ & $2 \cdot 25 b$ & $28 \cdot 16^{c}$ & 1.77 \\
\hline Week 8 & $12 \cdot 43^{\mathrm{a}}$ & 1.37 & $19 \cdot 94^{b}$ & $2 \cdot 67$ & $22 \cdot 41^{\mathrm{b}}$ & $1 \cdot 28 b$ & $31.01^{\mathrm{c}}$ & $2 \cdot 46$ \\
\hline
\end{tabular}

\section{Protein levels of key genes in hepatic and ileal lipid metabolism}

Expressions of hepatic protein (CYP7A1, HMG-CoAr and LDLR) and ileal protein (ASBT and IBABP) in the NC, BC and HD groups after 8-week feeding are shown in Fig. 4. Similar to the RT-PCR results, the expression levels of CYP7A1 and LDLR in the HD group were significantly higher, whereas the levels of HMG-CoAr and ASBT were significantly lower than in the BC group.

\section{Discussion}

To provide insights into the hypocholesterolaemic effect and mechanism of WHLB so as to offer economic significance for its consumption, the hypocholesterolaemic effect of WHLB was investigated systematically in the present study. The 2015-2020 Dietary Guidelines for Americans recommend that at least one-half of all grains consumed should be whole grains ${ }^{(33)}$. A growing body of evidence supports that increased whole-grain consumption is associated with a reduced risk of developing chronic diseases ${ }^{(15)}$. Therefore, we replaced all maize starch with WHLB (48.95\%) in the HD group. As a control, the LD group was fed with $10 \%$ WHLB, which was lower than the recommended dose. Our previous study showed that WHLB increased body weight and feed efficiency ratios while decreased the obesity degree of HFD rats ${ }^{(21)}$. Consistently, results of organ index tests showed that the indexes of liver and abdominal fat in HFD rats decreased significantly after administration of high-dose WHLB. The liver plays a central role in maintaining lipid homoeostasis ${ }^{(11)}$, and increased abdominal fat has been linked to increased CVD risk $^{(34)}$. These results suggested that WHLB regulated lipid metabolism.

The results of plasma and liver lipid tests showed that administration of high-dose WHLB significantly decreased the TC and LDL-cholesterol levels in the plasma and the TC and the 
Week 4

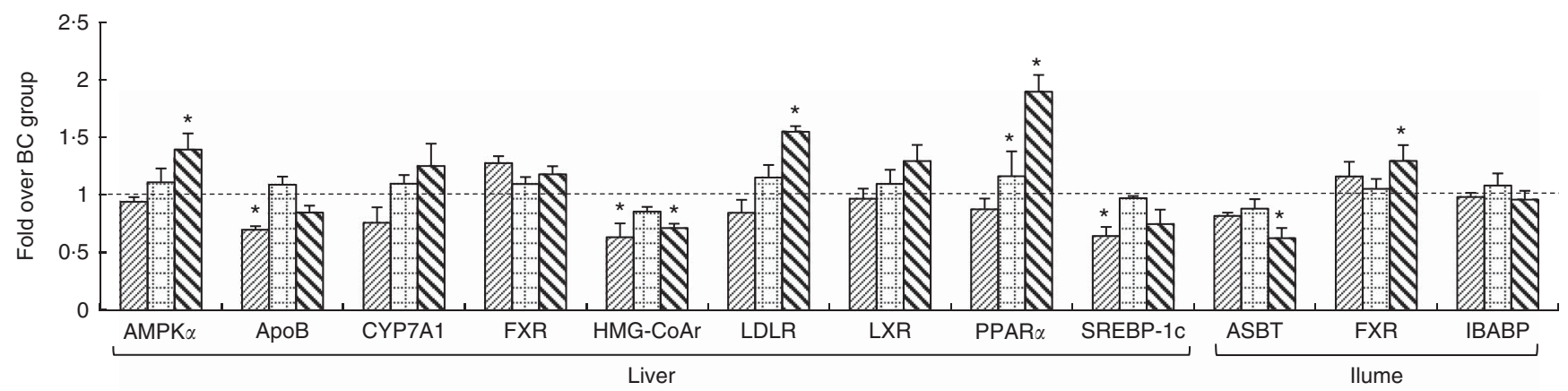

Week 8

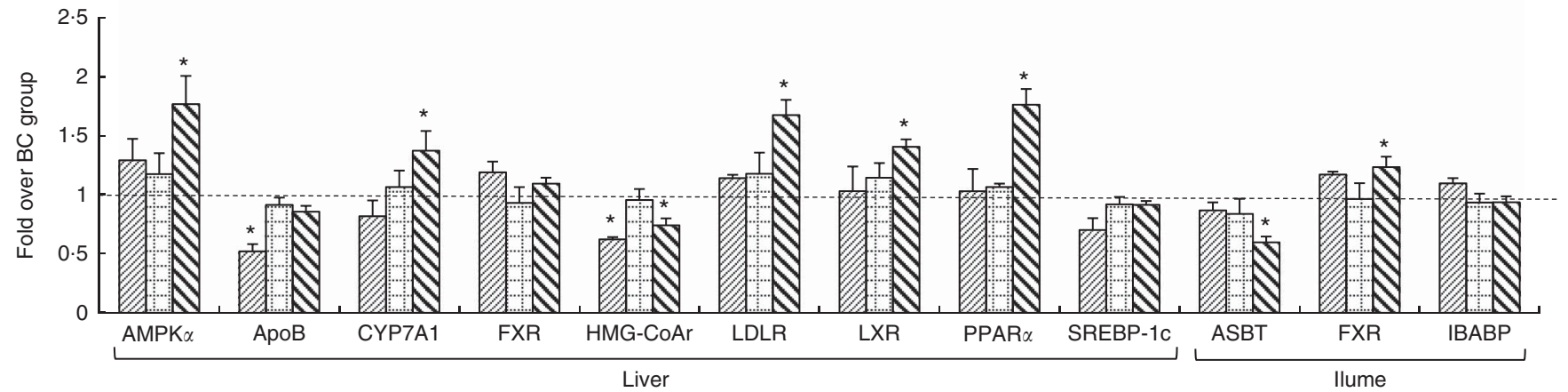

Fig. 3. mRNA levels of hepatic and ileal lipid metabolic regulators. Values are means $(n 9)$ and standard deviations. Each mRNA was normalised to $\beta$-actin and is expressed as a relative level to blank control (BC) group. ${ }^{*}$ mRNA levels of regulators were significantly different $(P<0.05)$ compared with the BC group. AMPKa, AMPactivated protein kinase $a$; CYP7A1, cholesterol 7a-hydroxylase; FXR, farnesoid X receptor; HMG-CoAr, 3-hydroxy-3-methylglutaryl coenzyme A reductase; LDLR, LDL receptor; LXR, liver X receptor; SREBP-1c, sterol regulatory element-binding protein-1c; ASBT, apical sodium-dependent bile acid transporter; IBABP, ileal bile

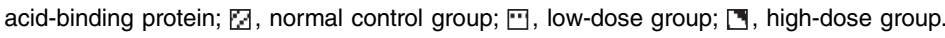

(A)

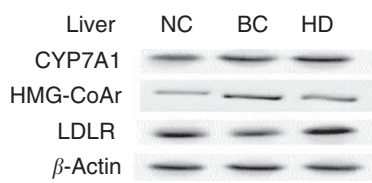

(B)

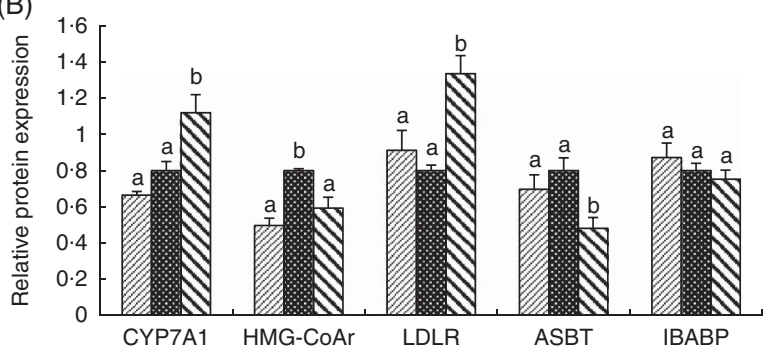

Fig. 4. Effect of whole-grain highland hull-less barley on hepatic protein (cholesterol 7a-hydroxylase (CYP7A1), 3-hydroxy-3-methylglutaryl coenzyme A reductase (HMG-CoAr) and LDL receptor (LDLR)) and ileal protein (apical sodiumdependent bile acid transporter (ASBT) and ileal bile acid-binding protein (IBABP)) expressions after 8 weeks of feeding. Values are means $(n 9)$ and standard deviations. The protein levels in each sample were normalised to the $\beta$-actin level. Histograms illustrate the densitometric analysis of protein levels shown in (A) based on $\beta$-actin expression (B). NC, normal control group; BC, blank control group; HD, high-dose group. $B$ : $⿴, N C ;, B C ; \quad H D$. ${ }^{a, b}$ Mean values of the same index in the three groups with unlike letters are significantly different $(P<0.05)$.

total lipid levels in the liver. Elevated plasma levels of TC and LDL-cholesterol are biomarkers for elevated risk of $\mathrm{CVD}^{(2)}$. Ishimwe et al. ${ }^{(35)}$ reported that $1 \%$ reduction in $\mathrm{TC}$ and $1 \%$ reduction in LDL-cholesterol translate to a $2 \%$ and $1 \%$ decrease in heart disease risk. The lipid-lowering activity of WHLB was consistent with previously evaluated barley ${ }^{(16,17,36)}$. The lipid regulation effect of WHLB was further confirmed through hepatocyte morphologic observation. The hepatic fat-droplet accumulation decreased after consuming WHLB diet, particularly at high doses. However, WHLB diet did not significantly affect TAG levels, which was in line with results from barley ${ }^{(16)}$ and oat diet ${ }^{(15)}$, whereas the mechanism should be further studied. HDL-cholesterol levels in the HD group were significantly higher than those in the BC group after 8-week feeding. High levels of functional HDL in plasma protect against atherosclerotic coronary disease ${ }^{(37)}$. Kim et al. ${ }^{(17)}$ reported that HDL-cholesterol levels in Syrian Golden hamsters with HFD were slightly increased after administrating diets that contain bread of whole-grain barley. Meanwhile, Sindhu \& Khetarpaul $^{(36)}$ reported that barley diet did not significantly affect HDL-cholesterol in mice. Hoang et al. ${ }^{(16)}$ reported that the HDLcholesterol levels were slightly decreased in HFD mice after a whole-grain barley diet. These differences may be attributed to the varieties of grains or experimental animal species used. Lipoprotein metabolism differs between different experimental model species. For example, mice show up to 40-fold higher LDL clearance by the liver compared with humans, and mice carry most of their plasma cholesterol in HDL particles ${ }^{(38)}$.

Cholesterol is mainly eliminated from the body via conversion to bile acids ${ }^{(16)}$. Bile acid levels in small intestinal contents 
and faeces were investigated accordingly. Results showed that diet with high-dose WHLB significantly increased the bile acid levels in small intestinal contents and faeces of HFD rats. These results suggested that WHLB may regulate lipid metabolism by increasing bile acid excretion, which is consistent with the mechanism of barley ${ }^{(16,39,40)}$. $\beta$-Glucan and dietary fibre compounds were reported to delay bile acid reabsorption and facilitate faecal bile acid excretions ${ }^{(17-19)}$. Martinez-Floresa et $a l^{(41)}$ reported that hypocholesterolaemic effect of dietary fibre attributed primarily to soluble dietary fibre. WHLB used in the current study has relatively high content of $\beta$-glucans and dietary fibre, particularly soluble dietary fibre, compared with other grains ${ }^{(42-44)}$. Therefore, the hypocholesterolaemic effect of WHLB may be partly attributed to its high content of $\beta$-glucan and (soluble) dietary fibre. Undigested dietary fibre will be fermented in the caecum, which is a site of vigorous microbial activity in rats, thereby yielding various products ${ }^{(45)}$. The values of caecum indexes and faecal dry weights in HD groups were significantly higher, whereas the faecal $\mathrm{pH}$ was markedly lower compared with those in the $\mathrm{BC}$ group, thereby corresponding to the changes in bile acid.

To study the molecular mechanism by which WHLB affects the lipid metabolism of the HFD rats, the mRNA levels of key genes in hepatic and ileal lipid metabolic were measured. AMPK $\alpha$, HMG-CoAr and SREBP-1c were proven to be involved in hepatic cholesterol synthesis. AMPK regulates lipid metabolism and inhibits cholesterol synthesis by inducing the inhibitory phosphorylation of HMG-CoAr. AMPK can also inhibit fatty acid synthesis by inducing the inhibitory phosphorylation of SREBP-1c ${ }^{(4)}$. HMG-CoAr is the rate-limiting enzyme for cholesterol synthesis ${ }^{(5)}$. Meanwhile, SREBP-1c is a transcription factor that modulates the expression of a large number of genes involved in the synthesis of cholesterol ${ }^{(6)}$. RT-PCR results showed that the mRNA levels of HMG-COAr and SREBP-1c were significantly up-regulated in the $\mathrm{BC}$ group, which indicated that HFD stimulated cholesterol synthesis. These results were corresponded with the high levels of plasma and liver lipid of the $\mathrm{BC}$ group. After administration of high-dose WHLB, the mRNA levels of HMG-CoAr significantly decreased, whereas the AMPK $\alpha$ levels significantly increased. These results suggested that the low TC concentrations in the HD group may be due to the downregulation of HMG-CoAr, which in turn may be partly caused by the up-regulation of AMPK $\alpha$. The down-regulation of HMG-CoAr after WHLB intake was also noted with the intake of barley ${ }^{(16)}$.

When the hepatic cholesterol synthesis increased, the liver secretes cholesterol with ApoB and increases the circulating $\mathrm{LDL}^{(7)}$. Increased level of LDL, which is the principal atherogenic lipoprotein in the blood, promotes cholesterol accumulation in the artery wall, thereby initiating atherosclerosis ${ }^{(46)}$. Therefore, the significant up-regulation of $\mathrm{ApoB}$ in the $\mathrm{BC}$ group may be responsible for the high LDL-cholesterol levels and abdominal fat indexes. Conversely, LDLR mediates endocytosis of LDL and increases the reabsorption of LDL, decreasing synthesis of liver cholesterol ${ }^{(8,9)}$. The significant upregulation of LDLR in the HD group may be responsible for the low LDL-cholesterol levels and abdominal fat indexes. HDL plays a central role in transporting cholesterol from extrahepatic tissues to the liver for catabolism of cholesterol to bile acids.
Thus, HDL is thought to contribute to removing cholesterol from peripheral tissues ${ }^{(11)}$. Regulator of HDL biogenesis is upregulated by the transcription factor LXR, and HDL expression is further enhanced by PPAR $\alpha$ activation ${ }^{(11)}$. Compared with the BC group, the significantly higher mRNA levels of PPAR $\alpha$ in the LD group may be responsible for the significantly lowered abdominal fat indexes at week 4 . The mRNA levels of LXR and PPAR $\alpha$ in the HD group were higher than those in the BC group, and the difference became significant after 8-week feeding. Consistent with our results, Ogata et al. ${ }^{(11)}$ concluded that PPAR and LXR are involved in HDL biogenesis in a cooperative signal transduction pathway. The concurrent upregulation of LXR and PPAR $\alpha$ may be responsible for the high HDL-cholesterol levels in the HD group.

In the liver, CYP7A1 catalyses the rate-limiting step in the bile acid synthetic pathway ${ }^{(47)}$. The mRNA levels of CYP7A1 in the HD group were significantly higher than those in the BC group after 8-week feeding. Musso et $a l^{(47)}$ reported that LXR can increase CYP7A1 transcription; therefore, the CYP7A1 upregulation may be attributed to LXR up-regulation. Consistent with our results, Choi et al. ${ }^{(48)}$ and Yang et al. ${ }^{(49)}$ reported that barley $\beta$-glucan diet significantly increased the mRNA level of CYP7A1. Hoang et al. ${ }^{(16)}$ reported that hepatic CYP7A1 expression was surprisingly decreased after barley diet, and they partly attributed the effect to the up-regulation of hepatic FXR. FXR, a ligand-activated transcription factor belonging to the adopted orphan receptor, plays an important role in maintaining the health of the liver and the intestines ${ }^{(50)}$. Hepatic FXR has been proposed to play a central role in the feedback repression of the CYP7A1 gene ${ }^{(12)}$. However, the negative correlation between FXR and CYP7A1 was uncertainty ${ }^{(28)}$. Conversely, Musso et al. ${ }^{(47)}$ reported that FXR activates the bile acid synthesis by inducing CYP7A1. Bile acids are reabsorbed by the ASBT at the end of the small intestine (ileum part) and transferred from the apical to the basolateral membrane by the IBABP $^{(13)}$. FXR was coupled to reduce ASBT expression, thereby resulting in decreased intestinal absorption of bile acids $^{(16)}$. ASBT is almost exclusively expressed in the terminal section of the ileum ${ }^{(51)}$. Therefore, the mRNA levels of ASBT, FXR and IBABP in the ileum were investigated. The significantly decreased level of ASBT after a diet with a high dose of WHLB, which may be caused by the significantly increased level of ileal FXR, was consistent with the reported effect of barley diet by Hoang et $a l^{(16)}$. Therefore, the CYP7A1 up-regulation and ASBT down-regulation may be responsible for the high content of bile acid in the small intestine and faeces after WHLB diet.

We only investigated the protein levels of CYP7A1, HMGCOAr, LDLR, ASBT and IBABP because of the limited mature experimental antibody. The protein levels of these biomarkers were consistent with their mRNA levels, thereby confirming the validity of the RT-PCR analysis. We only studied the mRNA and protein levels of several key genes related to hepatic and ileal lipid metabolism. However, the lipid regulatory mechanism of diet involves numerous proteins ${ }^{(52)}$. Therefore, proteome analysis should be performed in future studies to comprehensively investigate the lipid regulatory mechanism of WHLB.

In conclusion, high dose of WHLB has a hypocholesterolaemic effect, and the molecular mechanism of this effect may 
include inhibition of cholesterol synthesis through upregulation of AMPK $\alpha$ expression and down-regulation of HMG-CoAr expression; stimulation of cholesterol conversion to bile acids through up-regulation of CYP7A1 expression; decrease cholesterol accumulation in peripheral tissues through up-regulation of expression of LDLR, LXR and PPAR $\alpha$; inhibition of bile acid reabsorption through up-regulation of ileal FXR expression and down-regulation of ASBT expression

\section{Acknowledgements}

This work was financially supported by the Science and Technology Support Demonstration Project of Chongqing (CSTC2014JCSF-JCSSX004).

Contributions of authors are as follows: X. X. and J. K. designed the study; X. X., J. S. and G. L. performed the experiments; G. L. and J. Z. analysed the data; X. X. and G. L. wrote and revised the manuscript. All authors reviewed the manuscript.

The authors declare that there are no conflicts of interest.

\section{Supplementary material}

For supplementary material/s referred to in this article, please visit https://doi.org/10.1017/S0007114518000831

\section{References}

1. Seo HS \& Choi MH (2015) Cholesterol homeostasis in cardiovascular disease and recent advances in measuring cholesterol signatures. J Steroid Biochem Mol Biol 153, 72-79.

2. Shao DY, Bartley GE, Yokoyama W, et al. (2013) Plasma and hepatic cholesterol-lowering effects of tomato pomace, tomato seed oil and defatted tomato seed in hamsters fed with high-fat diets. Food Chem 139, 589-596.

3. Zhang L, Zhou M, Fang GS, et al. (2013) Hypocholesterolemic effect of capsaicinoids by increased bile acids excretion in ovariectomized rats. Mol Nutr Food Res 57, 1080-1088.

4. Jeon SM (2016) Regulation and function of AMPK in physiology and diseases. Exp Mol Med 48, e245.

5. Chen X, Wang XF, Li ZJ, et al. (2012) Molecular cloning, tissue expression and protein structure prediction of the porcine 3-hydroxy-3-methylglutaryl-coenzyme A reductase (HMGR) gene. Gene 495, 170-177.

6. Ji JY, Zhao XH, Leng L, et al. (2011) Comparison of dietary control and atorvastatin on high fat diet induced hepatic steatosis and hyperlipidemia in rats. Lipids Health Dis 10, 23.

7. Kim S, Hong J, Jeon R, et al. (2016) Adzuki bean ameliorates hepatic lipogenesis and proinflammatory mediator expression in mice fed a high-cholesterol and high-fat diet to induce nonalcoholic fatty liver disease. Nutr Res 36, 90-100

8. Kemper MF, Srivastava S., King MT, et al. (2015) An ester of $\beta$-Hydroxybutyrate regulates cholesterol biosynthesis in rats and a cholesterol biomarker in humans. Lipids 50, 1185-1193.

9. Levinson SS \& Wagner SG (2015) Implications of reverse cholesterol transport: Recent studies. Clin Chim Acta 439, 154-161.

10. He WS, Wang MG, Pan XX, et al. (2013) Role of plant stanol derivatives in the modulation of cholesterol metabolism and liver gene expression in mice. Food Chem 140, 9-16.

11. Ogata M, Tsujita M, Hossain MA, et al. (2009) On the mechanism for PPAR agonists to enhance ABCA1 gene expression. Atherosclerosis 205, 413-419.
12. Moschetta A (2015) Nuclear receptors and cholesterol metabolism in the intestine. Atheroscler Suppl 17, 9-11.

13. Fu ZD \& Klaassen CD (2013) Increased bile acids in enterohepatic circulation by short-term calorie restriction in male mice. Toxicol Appl Pharmacol 273, 680-690.

14. Ross BA, Bruce SJ, Blondel-Lubrano A, et al. (2011) A wholegrain cereal-rich diet increases plasma betaine, and tends to decrease total and LDL-cholesterol compared with a refinedgrain diet in healthy subjects. Br J Nutr 105, 1492-1502.

15. Zhou AL, Hergert N, Rompato G, et al. (2015) Whole grain oats improve insulin sensitivity and plasma cholesterol profile and modify gut microbiota composition in C57BL/6J Mice. J Nutr 145, 222-230.

16. Hoang MH, Houng SJ, Jun HJ, et al. (2011) Barley intake induces bile acid excretion by reduced expression of intestinal ASBT and NPC1L1 in C57BL/6J Mice. J Agric Food Chem 59, 6798-6805.

17. Kim H, Turowski M, Anderson WHK, et al. (2011) Supplementation of hydroxypropyl methylcellulose into yeast leavened all-whole grain barley bread potentiates cholesterollowering effect. J Agric Food Chem 59, 7672-7678.

18. Delaney B, Nicolosi RJ, Wilson TA, et al. (2003) $\beta$-Glucan fractions from barley and oats are similarly antiatherogenic in hypercholesterolemic Syrian golden hamsters. J Nutr 133, 468-495.

19. De Angelis M, Montemurno E, Vannini L, et al. (2015) The role of whole-grain barley on human fecal microbiota and metabolome. Appl Environ Microbiol 81, 7945-7956.

20. Knutsen SH \& Holtekjølen AK (2007) Preparation and analysis of dietary fibre constituents in whole grain from hulled and hull-less barley. Food Chem 102, 707-715.

21. Xia XX, Li GN, Ding YB, et al. (2017) Effect of whole grain Qingke (Tibetan Hordeum vulgare L. Zangqing 320) on the serum lipid levels and intestinal microbiota of rats under highFat diet. J Agric Food Chem 65, 2686-2693.

22. American Association of Cereal Chemists (2003) Approved Method of the AACC, 10th ed. St. Paul, MN: AACC.

23. Association of Official Analytical Chemists (1980) Official Method of Analysis of AOAC Intl, 13th ed. Arlington, VA: AOAC.

24. Reeves PG, Nielsen FH \& Fahey GC (1993) AIN-93 purified diets for laboratory rodents: final report of the American Institute of Nutrition ad hoc writing committee on the reformulation of the AIN-76A rodent diet. J Nutr $\mathbf{1 2 3}$, 1939-1951.

25. Charan J \& Kantharia ND (2013) How to calculate sample size in animal studies? J Pharmacol Pharmacother 4, 303-306.

26. Institute of Laboratory Animal Resources, National Research Council (US) \& Institute for Laboratory (1985) Guide for the Care and Use of Laboratory Animals. Washington, DC: National Academies Press.

27. Zhang L, Fang GS, Zheng LH, et al. (2013) Hypocholesterolemic effect of capsaicinoids in rats fed diets with or without cholesterol. J Agric Food Chem 61, 4287-4293.

28. Park SO \& Park BS (2015) Bifidogenic effect of grain larvae extract on serum lipid, glucose and intestinal microflora in rats. $J$ Biosci $\mathbf{4 0}, 513-520$.

29. Liu X, Ogawa H, Kishida T, et al. (2010) The effect of high-amylose cornstarch on lipid metabolism in OVX rats is affected by fructose feeding. J Nutr Biochem 21, 89-97.

30. Folch J, Lees M \& Sloane Stanley G (1957) A simple method for the isolation and purification of total lipides from animal tissues. J Biol Chem 226, 497-509.

31. Zhang L, Zheng LH, Tian BM, et al. (2013) Effects of capsaicinoids on cholesterol metabolism and its related gene. Acta Nutrimenta Sinica 35, 381-386 (In Chinese with English abstract). 
32. Xia XJ, Ran CX, Ye XJ, et al. (2017) Monitoring of the bacterial communities of bamboo shoots (Dendrocalamus latiflorus) during pickling process. Int J Food Sci Tech 52 , $1101-1110$.

33. US Department of Agriculture (2015) Dietary guidelines for Americans, 2015-2020. https://health.gov/dietaryguidelines/ 2015/guidelines/ (accessed November 2017).

34. Carmina E, Bucchieri S, Esposito A, et al. (2007) Abdominal fat quantity and distribution in women with polycystic ovary syndrome and extent of its relation to insulin resistance. J Clin Endocrinol Metab 92, 2500-2505.

35. Ishimwe N, Daliri EB, Lee BH, et al. (2015) The perspective on cholesterol-lowering mechanisms of probiotics. Mol Nutr Food Res 59, 94-105.

36. Sindhu SC \& Khetarpaul K (2003) Effect of feeding probiotic fermented indigenous food mixture on serum cholesterol levels in mice. Nutr Res 23, 1071-1080.

37. Leiva A, Verdejo H, Benítez ML, et al. (2011) Mechanisms regulating hepatic SR-BI expression and their impact on HDL metabolism. Atherosclerosis 217, 299-307.

38. van der Wulp MYM, Verkade HJ \& Groen AK (2013) Regulation of cholesterol homeostasis. Mol Cell Endocrinol 368, 1-16.

39. Dongowskia G, Hutha M \& Gebhardta E (2003) Steroids in the intestinal tract of rats are affected by dietary-fibre-rich barleybased diets. Br J Nutr 90, 895-906.

40. Zhong YD, Marungruang N \& Fåk F (2015) Effects of two whole-grain barley varieties on caecal SCFA, gut microbiota and plasma inflammatory markers in rats consuming low- and high-fat diets. Br J Nutr 113, 1558-1570.

41. Martinez-Floresa HE, Chang YK, Martinez-Bustos F, et al. (2004) Effect of high fiber products on blood lipids and lipoproteins in hamsters. Nutr Res 24, 85-93.

42. Yalçın E, Çelik S, Akar T, et al. (2007) Effects of genotype and environment on $\beta$-glucan and dietary fiber contents of hull-less barleys grown in Turkey. Food Chem 101, 171-176.
43. Andersson AAM, Andersson R, Piironen V, et al. (2013) Contents of dietary fibre components and their relation to associated bioactive components in whole grain wheat samples from the HEALTHGRAIN diversity screen. Food Chem 136, $1243-1248$

44. Zhang GP, Wang JM \& Chen JX (2002) Analysis of beta-glucan content in barley cultivars from different locations of China. Food Chem 79, 251-254.

45. Kishida T, Nogami H, Ogawa H, et al. (2002) The hypocholesterolemic effect of high amylose cornstarch in rats is mediated by an enlarged bile acid pool and increased fecal bile acid excretion, not by cecal fermented products. $J$ Nutr 132, 2519-2524.

46. Tall AR \& Yvan-Charvet L (2015) Cholesterol, inflammation and innate immunity. Nat Rev Immunol 15, 104-116.

47. Musso G, Gambino R \& Cassader M (2013) Cholesterol metabolism and the pathogenesis of non-alcoholic steatohepatitis. Prog Lipid Res 52, 175-191.

48. Choi JS, Kim H, Jung MH, et al. (2010) Consumption of barley $\beta$-glucan ameliorates fatty liver and insulin resistance in mice fed a high-fat diet. Mol Nutr Food Res 54, 1004-1013.

49. Yang JL, Kim YH, Lee HS, et al. (2003) Barley beta-glucan lowers serum cholesterol based on the up-regulation of cholesterol 7alpha-hydroxylase activity and mRNA abundance in cholesterol-fed rats. J Nutr Sci Vitaminol 49, 381-387.

50. Zhang XQ, Osaka T \& Tsuneda S (2015) Bacterial metabolites directly modulate farnesoid X receptor activity. Nutr Metab 12, $1-14$

51. Out C, Patankar JV, Doktorova M, et al. (2015) Gut microbiota inhibit Asbt-dependent intestinal bile acid reabsorption via Gata4. J Hepatol 63, 697-704.

52. Domínguez-Avila JA, Alvarez-Parrilla E, López-Díaz JA, et al. (2015) The pecan nut (Carya illinoinensis) and its oil and polyphenolic fractions differentially modulate lipid metabolism and the antioxidant enzyme activities in rats fed highfat diets. Food Chem 168, 529-537. 